\title{
On Leland's Option Hedging Strategy with Transaction Costs *
}

\author{
Yonggan Zhao ${ }^{\dagger}$ and William T. Ziemba
}

July 26,2003

JEL Classification: B23 C15 C61 G13

*Without implicating them, the authors are grateful to George Constantinides, Mark Davis, Yuri Kabanov and Harry Markowitz for helpful comments on an earlier draft. Zhao acknowledges financial support from Nanyang Business School, and Ziemba the Natural Sciences and Engineering Research Council of Canada.

${ }^{\dagger}$ Yonggan Zhao: Division of Banking and Finance, Nanyang Business School, Nanyang Technological University, Singapore, 639798, e-mail: aygzhao@ntu.edu.sg

${ }^{\ddagger}$ William T. Ziemba: Sauder School of Business, University of British Columbia, Canada, V6T 1Z2, e-mail: ziemba@interchange.ubc.ca 


\title{
On Leland's Option Hedging Strategy with Transaction Costs
}

\begin{abstract}
Nonzero transaction costs invalidate the Black-Scholes (1973) arbitrage argument based on continuous trading. Leland (1985) developed a hedging strategy which modifies the Black-Scholes hedging strategy with a volatility adjusted by the length of the rebalance interval and the rate of the proportional transaction cost. Leland claimed that the exact hedge could be achieved in the limit as the length of rebalance intervals approaches zero. Unfortunately, the main theorem (Leland 1985, P1290) is in error. Simulation results also confirm opposite findings to those in Leland (1985). Since standard delta hedging fails to exactly replicate the option in the presence of transaction costs, we study a pricing and hedging model which is similar to the delta hedging strategy with an endogenous parameter, namely the volatility, for the calculation of delta over time. With transaction costs, the optimally adjusted volatility is substantially different from the stock's volatility under the criterion of minimizing the mean absolute replication error weighted by the probabilities that the option is in or out of the money. This model partially explains the phenomenon that the implied volatilities with equity options are skewed. Data on S\&P500 index cash options from January to June 2002 are used to illustrate the model. Option prices from our model are highly consistent with the Black-Scholes option prices when transaction costs are zero.
\end{abstract}


LELAND (1985) DEVEloped A HEDGing STRATEGy that uses the Black and Scholes (1973) formula with a modified volatility dependent on the rate of transaction costs and the length of trading intervals. It is claimed that the modified strategy, inclusive of transaction costs, can be used to approximately replicate the option's payoff as the length of rebalance intervals becomes short. The idea was to offset the transaction costs by properly adjusting the volatility with respect to the length of trading intervals and develop a strategy that converges to the Black-Scholes price as transaction costs become arbitrarily small. Unfortunately, the main theorem (Leland 1985, P1290) is flawed. Intuitively, if the volatility is made arbitrarily large by making the length of rebalance intervals shorter, the hedging strategy converges to a trivial case which holds one share of the underlying stock at any point in time no matter how low the transaction cost rate is. As in Davis and Clark (1994), and Soner, Shreve, and Cvitanic (1995), this strategy confirms that the minimum cost for hedging a call option is exactly the price of the stock in the presence of transaction cost and in the framework of continuous trading. This strategy does not provide an exact hedge, since the payoff of holding the underlying stock is greater than that of the call option at maturity as long as the strike price of the option is positive.

Given that option premiums are determined by an optimal hedging strategy, the writer of a call option is interested in knowing when and how a hedging trade is triggered in the presence of transaction costs. Equity option prices are consistently higher (lower) than the Black-Scholes model prices for in- (out-of-) the-money options, as implied volatilities of in(out-of-) the-money options are higher (lower) than those of at-the-money ones. This market phenomenon has been documented as implied volatility skewness. Why would we see such a skewness? It is conceivable that trading frictions, including transactions costs, can partially be a reason. If the underlying stock prices are far above the strike price, the transactions costs should be large, therefore, the premiums of the options should be greater than the Black-Scholes model price.

Option replication has been extensively studied by numerous researchers. Besides Leland (1985), Boyle and Emanuel (1980) studied the distribution of hedging error across time and concluded that the hedging errors are uncorrelated. Boyle and Vorst (1992) designed a perfect 
hedging strategy in the Cox, Ross, and Rubinstein (1979) binomial model with transaction costs. The perfect hedge is possible due to the assumption of the binomial process for the underlying stock price. They also developed a similar risk neutral valuation approach that is a two state Markov process which greatly reduces the computational complexity. Based on Boyle and Vorst (1992), Edirisinghe, et al. (1993) developed a general replicating strategy in the framework of optimization by minimizing the initial cost subject the hedging portfolio payoff to being at least as large as the option's payoff. They indicated that it is not necessarily optimal to revise the portfolio at each revision points. On the other hand, if the replicating portfolio is allowed to dominate the option's payoff in some states, the minimum cost of this "super-replication" maybe less costly than the exact hedge. Toft (1996) studies the mean variance tradeoff in option replication. Assuming the option is priced with the Black-Scholes formula as in Leland (1985), Toft calculated the expected hedging errors and gives a closed form solution. Kabanov and Safarian (1997) gave a bound on the hedging error with proportional transaction costs and concluded that Leland's option hedging strategy is only a conjecture, but they did not uncover the error in the designed strategy. We use both simulation and mathematical arguments to disclaim the hedging strategy developed in Leland (1985).

Constantinides and Zariphopoulou $(1999,2001)$ examined the bounds for the prices of contingent claims in an intertemporal economy with transaction costs based on general preferences. As Beinsad et al. (1992) point out, there are no strategies that can perfectly replicate a contingent claim in the presence of transaction costs. In the study of option replication with transaction costs, there are two main issues that must be addressed. The first is how often the hedging portfolio should be rebalanced, and the second is how hedging errors can be minimized. These issues are in conflict. Frequent hedges reduce errors but augment costs, while less frequent hedges result in large errors. Although the statement in Leland (1985) is not correct, the idea is useful. The question is whether we can achieve some valuation approach such that the hedging error is minimized if an augmented volatility does not offset the transaction costs. This paper develops a pricing and hedging model based on portfolio replication techniques. Instead of using an exogenous augmented volatility, we 
endogenize the volatility, for the calculation of delta over time. We decide the optimally adjusted volatility through minimization of the mean absolute replication error. According to whether the option is in or out of the money, the total replication error is divided into two quantities, the upside error and the downside error, which are weighted by the probabilities that the option is in or out of the money. As the probability of the moneyness increases, the downside error is considered as more significant. Similarly, as the probability of the moneyness increases, the upside error is considered less significant.

This approach has the following features: i) the strategy is based on preference-domination instead of exact replication; ii) all candidate strategies are practically set to be based on the observation of changes of the underlying stock; and iii) the price of an option is also given, as in Leland (1985), by the Black-Scholes formula with the optimal volatility, which is endogenously determined with the agent's preference for risk and the optimal hedging strategy. Taking S\&P 500 cash options for a comparison, simulation results show that option prices for the suggested model are very close to the actual prices, especially for deep in-the-money and deep out-of-the-money options. This result is consistent with the volatility skewness. To test the accuracy of our model for option pricing, we carry out a calculation excluding transaction costs to see how close is our model to the Black-Scholes model, and we find that options prices are almost identical to each other through simulation.

Section I discusses dynamic hedging and uncovers the flaw in Leland (1985) using simulation and mathematical arguments. Section II presents an alternative model based on the minimization of the adjusted replication error based on the moneyness of the option. Section III tests the model via simulations using option data. Section IV concludes. 


\section{Simulation Comparisons and the Flaw}

\section{A. The Procedure of Dynamic Hedging}

Before we discuss Leland's work, we review dynamic hedging. Consider a market in which a security is traded with a proportional transaction cost rate $k$. Assume that an agent sells a derivative security for $C_{0}$ with a payoff $C_{T}$ depending only on the value of the underlying security at the expiration date $T$. The agent can use the amount $C_{0}$ to buy or sell the underlying asset in any amount to hedge such a financial contract without incurring any loss in any possible state at $T$. Let $S_{t}$ be the price dynamics of the underlying security and assume that it follows the lognormal process

$$
\frac{d S_{t}}{S_{t}}=r d t+\sigma d z_{t}
$$

where $z_{t}$ is a standard Brownian motion and $r$ is the interest rate. Hence, we confine our discussions of any statistical quantity in the risk neutral probability. The initial value of the hedging portfolio is

$$
P_{0}=C_{0}-k\left|\theta_{0}\right| S_{0}
$$

where $\theta_{0}$ is the initial holding of the underlying stock. At time $t$, the agent's portfolio is

$$
P_{t}=\theta_{t} S_{t}+B_{t}
$$

where $B_{t}$ is the amount in the bank account and $\theta_{t}$ is the number of shares held at time t. Assume the bank account earns a continuously compound rate of $r$ per annum for both borrowing and lending. The agent's goal is to maintain a portfolio that replicates the derivative's payoff $C_{T}$ with a dynamic trading strategy. In any time increment $\Delta t$, the change in portfolio value is

$$
P_{t+\Delta t}-P_{t}=\theta_{t}\left(S_{t+\Delta t}-S_{t}\right)+\left(B_{t} e^{r \Delta t}-B_{t}\right)-k\left|\theta_{t+\Delta t}-\theta_{t}\right| S_{t+\Delta t}
$$

In Equation (2), the first term on the right hand side represents the profit/loss due to the change in the value of the underlying security, the second is the interest paid or received from the bank account, and the third is the transaction cost of trading. 
The portfolio $P_{t}$ is required to replicate the value of the derivative security $C\left(S_{t}, t\right)$ which is a function of the value of the underlying stock and time. When $k=0$ (no transaction costs), we can derive the Black-Scholes partial differential equation by letting the length of the rebalance interval approach zero and applying Itô's formula. The partial differential equation that $C(S, t)$ satisfies and the number of shares, $\theta_{t}$, held in the stock are

$$
\left\{\begin{array}{l}
-r C+\frac{\partial C}{\partial t}+r S \frac{\partial C}{\partial S}+\frac{1}{2} S^{2} \sigma^{2} \frac{\partial^{2} C}{\partial S^{2}}=0 \\
\theta_{t}=\frac{\partial C}{\partial S} .
\end{array}\right.
$$

For a call option struck at $K$, the terminal payoff is $C_{T}=\max \left(S_{T}-K, 0\right)$ and the solution to $(3)$ is given by the Black-Scholes formula

$$
C\left(S_{t}, t\right)=S_{t} N\left(d_{t}\right)-K e^{-r(T-t)} N\left(d_{t}-\sigma \sqrt{T-t}\right)
$$

where $N(\cdot)$ is the standard normal cumulative function and

$$
d_{t}=\frac{\ln \frac{S_{t}}{K}+\left(r+\frac{1}{2} \sigma^{2}\right)(T-t)}{\sigma \sqrt{T-t}} .
$$

Remark. As we will show, with transaction cost $k \neq 0$, a similar partial differential equation approach is not available due to the dependence of $\left|\theta_{t+\Delta t}-\theta_{t}\right|$ on the change of $\Delta t$. However, there is some research, for example Hoggard et al.(1994), which claims that a similar PDE with an adjusted volatility can be derived. The related pricing procedure is only an approximation, since solution to the associated partial differential equation,

$$
\left\{\begin{array}{l}
-r C+\frac{\partial C}{\partial t}+r S \frac{\partial C}{\partial S}+\frac{1}{2} S^{2} \hat{\sigma}^{2} \frac{\partial^{2} C}{\partial S^{2}}=0 \\
C(S, T)=(S-K)^{+}
\end{array}\right.
$$

is not the original option's price but it is the price of a call option with underlying security price dynamics

$$
\frac{d \hat{S}_{t}}{\hat{S}_{t}}=\mu d t+\hat{\sigma} d z
$$

where $\hat{\sigma}$ is the augmented (hedging) volatility as in Leland (1985). 


\section{B. Simulation Results and Comparisons}

We present some simulation results to help understand the effect of transaction costs in option hedging. Suppose the underlying stock has a volatility $25 \%$ and the interest rate is $5 \%$ per annum. Let $\Delta t=1 / 260,1 / 520,1 / 1040,1 / 4160,1 / 8320$, with initial value $S_{0}=100$. We first carry out replications of call options with strike prices $K=80,90,100,110,120$ for the case of no transaction cost. The hedging strategy is devised as in (4). Table I presents the results based on an analysis of 1000 scenarios.

$$
<<\text { Table I about here }>>
$$

At any time $t$, the portfolio holds $N\left(d_{t}\right)$ shares which are financed through a bank account paying annual rate $r$. By examining Table I, we find that the options are approximately hedged as the rebalance intervals become sufficiently short. With a rebalance on a daily base, the differences between the terminal values of the hedging portfolios and the payoffs of the options at expiration have the expected values within one-hundredth with standard devia-

tions below 0.65. Furthermore, as the rebalance time shrinks, say $\Delta t=\frac{1}{8320}$, the means of those differences are accurate to the nearest one-thousandth with standard deviations ranging from 0.05 to 0.11 . Figure 1 depicts the scenario distribution of the difference between the hedging portfolio value and the option's terminal payoff for a particular parameter setting.

$$
<<\text { Figure } 1 \text { about here }>>
$$

Generally, Table I and Figure 1 show that replication is implementable under the assumption of no transaction costs.

However, replication can become chaotic when transactions costs are present. Leland (1985) suggested an augmented volatility in the Black-Scholes formula to offset the hedging errors caused by the transaction costs. In his paper, the adjusted volatility $\hat{\sigma}$ was defined as

$$
\hat{\sigma}=\sigma \sqrt{1+\sqrt{\frac{2}{\pi}} \times \frac{k}{\sigma \sqrt{\Delta t}}} .
$$


Since increasing volatility enlarges option's premium, the idea of introducing an augmented volatility is to offset the necessary transaction costs from hedging by using the extra option premium. However, the adjusted delta-hedging does not converge to the payoff of the original option. For transaction cost rate $k=0.001$, Table II presents the simulation results for the same parameter setting as in the case of no transaction costs.

$$
<<\text { Table II about here }>>
$$

The hedging errors are not negligible, contrary to Leland's result. Hedging on a daily base can introduce mean errors ranging from -0.1819 to -0.2982 with standard deviations from 0.3077 to 0.6551 . As rebalancing becomes finer, the hedging errors increase rapidly across different strike prices . For example, when $\Delta t=1 / 8320$, mean hedging errors range from -0.7486 to -1.4584 with standard deviations ranging from 0.4285 to 0.6531 across different strike prices. Figure 2 depicts the scenario distribution of the difference between the hedging portfolio value and the option's terminal payoff for a particular parameter setting.

$$
<<\text { Figure } 2 \text { about here }>>
$$

Compared to the case of no transaction costs, the options' premiums are larger, but hedging errors are not able to be eliminated by the extra premiums. The hedging errors stay not only in terms of expectation, but also in large standard deviations. What has gone wrong? Obviously, the hedging strategy designed in Leland (1985) does not hedge the original option in question but something else.

\section{The Leland Model}

As a widely cited research paper on option hedging and pricing with transaction costs, Leland (1985) introduced a theory that modifies the Black-Scholes formula with an augmented volatility to circumvent transaction costs . Leland defined the augmented volatility $\hat{\sigma}$ as in (5) and

$$
\hat{C}\left(S_{t}, t ; K, \sigma^{2}, r, T, k, \Delta t\right)=S_{t} N\left(\hat{d}_{t}\right)-K e^{-r(T-t)} N\left(\hat{d}_{t}-\hat{\sigma} \sqrt{T-t}\right)
$$


where

$$
\hat{d}_{t}=\frac{\ln \frac{S_{t}}{K}+\left(r+\frac{1}{2} \hat{\sigma}^{2}\right)(T-t)}{\hat{\sigma} \sqrt{T-t}} .
$$

That is, $\hat{C}$ is the Black-Scholes option price based on the modified volatility (5). Since the augmented volatility does not depend on the strike price, one might be skeptical about the truth of the theory in the first place.

Adopting the modified delta hedging, he "proved" the following theorem:

Theorem (Leland 1985, P1290). Following the modified delta hedging strategy, the Black-Scholes price $\hat{C}$ will yield $\max \left(S_{T}-K, 0\right)$ almost surely inclusive of transactions costs, as $\Delta t \rightarrow 0$.

In the "proof" of the theorem, the delta hedging strategy was defined as: holding $\hat{C}_{S}$ shares of the underlying security and invest $\hat{C}-\hat{C}_{S} S$ in the bank account. The following expression of the hedging error is examined

$$
\Delta H=\Delta P-\Delta \hat{C}-T C
$$

with

$$
\begin{aligned}
& \Delta P=\hat{C}_{S} S\left(\frac{\Delta S}{S}\right)+\left(\hat{C}-\Delta C_{S} S\right) r \Delta t+O\left(\Delta t^{2}\right) \\
& \Delta \hat{C}=\hat{C}_{S} S\left(\frac{\Delta S}{S}\right)+\frac{1}{2} \hat{C}_{S S} S^{2}\left(\frac{\Delta S}{S}\right)^{2}+\hat{C}_{t} \Delta t+O\left(\Delta t^{3 / 2}\right) \\
& T C=\frac{1}{2} k \hat{C}_{S S} S^{2}\left|\frac{\Delta S}{S}\right|+O\left(\Delta t^{3 / 2}\right)
\end{aligned}
$$

where $T C$ is the transaction cost from time $t$ to $t+\Delta t$. By showing that the total hedging error

$$
H=\sum_{t=0}^{T-\Delta t} \Delta H_{t} \rightarrow 0, \quad \text { as } \quad \Delta t \rightarrow 0
$$

the proof is completed 1

\footnotetext{
${ }^{1}$ Leland's footnotes 10 and 11 also include a mathematical error. The following statement is not true in general

$$
\hat{d} \sim O\left(\Delta t^{-\frac{1}{4}}\right) \Rightarrow \exp \left(-\frac{1}{2} \hat{d}^{2}\right) \sim O\left(\exp \left(-\frac{1}{2} \Delta t^{-\frac{1}{2}}\right)\right) .
$$

For example, if $\hat{d}=\frac{1}{2} \Delta t^{-\frac{1}{4}}$, then $\hat{d} \sim O\left(\Delta t^{-\frac{1}{4}}\right)$, But

$$
\exp \left(-\frac{1}{2} \hat{d}^{2}\right) \nsim O\left(\exp \left(-\frac{1}{2} \Delta t^{-\frac{1}{2}}\right)\right)
$$
}


The portfolio defined was not self-financing. Actually, it was implicitly assumed that the portfolio value $P_{t}$ is equal to $\hat{C}\left(S_{t}, t\right)$ at any time $t$, which is not correct. Since we hope to derive that the hedging portfolio, therefore the option's price, is given by such a formula $\hat{C}$, we cannot prove a statement that is based on the truth of the statement. Although the hedging portfolio can start with $\hat{C}$, but the values at later stages may deviate due to transaction costs.

The increment of the hedging portfolio value may be properly written as

$$
\Delta P=\hat{C}_{S} S\left(\frac{\Delta S}{S}\right)+\left(\hat{C}-\hat{C}_{S} S\right) r \Delta t+\left(P_{t}-\hat{C}\right) r \Delta t+O\left(\Delta t^{2}\right)
$$

The hedging error is

$$
\Delta H=\frac{1}{2} \hat{C}_{S S} S^{2}\left[\sigma^{2} \Delta t-\left(\frac{\Delta S}{S}\right)^{2}+k\left[E\left|\frac{\Delta S}{S}\right|-\left|\frac{\Delta S}{S}\right|\right]\right]+\left(P_{t}-\hat{C}\right) r \Delta t+O\left(\Delta t^{3 / 2}\right) .
$$

The first term in the above equation is exactly the same as in Leland's paper which converges to zero in probability. The second term is unknown. It is only possible that the sum of these terms over all rebalance intervals approaches zero if each $P_{t}-\hat{C}$ approaches zero uniformly in $t$ as $\Delta t \rightarrow 0$. Hence, it is not guaranteed that the total hedging error $\sum_{t=0}^{T-\Delta t} \Delta H$ approaches zero as $\Delta t \rightarrow 0$.

The error that ruins the technique of this replicating strategy is a flawed mathematical argument. In Leland's footnotes 10 and 11, the statement, $\hat{d}_{t} \sim O\left(\Delta t^{-\frac{1}{4}}\right)$, was used to prove that $\frac{\partial^{2} C}{\partial S^{2}}$ and $\frac{\partial^{2} C}{\partial S \partial t}$ are $O\left(\Delta t^{\frac{1}{2}}\right) . \hat{d}_{t} \sim O\left(\Delta t^{-\frac{1}{4}}\right)$ was derived using $\hat{d}_{1} \rightarrow \frac{1}{2} \hat{\sigma}(T-t)^{1 / 2}$, which is not true for all $t<T$ uniformly. Consider the period closest to the expiration date, i.e., $t=T-\Delta t . \hat{\sigma}(T-t)^{1 / 2}$ becomes arbitrarily small, but it does not diverge to infinity as required. To complete the proof of the theorem, a "uniform" convergence of $\hat{d}_{t}$ to $O\left(\Delta t^{-1 / 4}\right)$ is required which is not true. The variability of $\frac{\partial^{2} C}{\partial S^{2}}$ is chaotic when the option is close to expiration, because $N^{\prime}\left(d_{t}\right)$ can not guarantee to become arbitrarily small uniformly in $t$ as $\Delta t$ approaches zero.

It is also interesting to examine the limiting case. Since $\hat{\sigma}$ goes to infinity as $\Delta t \rightarrow 0$, the option's price approaches the value of the underlying security. The hedging portfolio will be trivial in the limiting case: holding one share of the underlying security, hence, the 
transaction cost is zero. That is, the initial option's value should be equal to the value of the underlying security, and the hedging portfolio will never exactly be the same as the payoff of the option as long as the option's strike price $K>0$, in contrast with Footnote 13 in Leland (1985).

\section{A Pricing and Hedging Model}

\section{A. Implied Volatilities and the Hedging Strategies}

Market prices of options are usually higher (lower) than the Black-Scholes prices for in (out of) the money call options. This market imperfection has been documented as volatility skew as in Rubinstein (1994). Standard option pricing theory suggests that the implied volatilities for all option contracts with different strikes should be flat in an orderly market. However, evidence from the equity option market has shown that implied volatilities are heavily skewed. With $18.42 \%$ and $1.7 \%$ as the estimated volatility of the index and the short interest rate, respectively, Figure 3 depicts the implied volatilities for S\&P 500 cash options with expiration in June 2002, using data from January 1 to June 20, 2002.

$$
<<\text { Figure } 3 \text { about here }>>
$$

It is not clear whether there is a systematic pricing rule better than the Black-Scholes formula in the presence of transaction costs. If alternative pricing approaches are not able to be sought, why not consider a method that is intuitive as the Black-Scholes model? It is Leland (1985) who first considered this idea: The increment of the option premium induced by an augmented volatility can offset the necessary transaction costs. With the Black-Scholes formula modified with an augmented volatility, the delta hedging strategy was expected to "exactly" replicate the options payoff in the limit. One might be doubtful about the setting of the augmented volatility as in (5), since it does not depend on the option's strike price $K$. The skewness of the implied volatilities across various strike prices suggests that, if such an adjusted volatility can do the job, it must be dependent on the option's strike price. 
It can be proved that, as Leland's augmented volatility for the $\Delta$ hedging increases to infinity (which is the case as the length of the rebalance interval approaches zero), the hedging portfolio will eventually hold one share of the underlying security throughout the horizon, which reduces the transaction costs but increases the hedging error. On the other hand, if frequency of hedging is increased, the hedging error will be small but the cost will be enormous, resulting in an increment of the option's premium. How should we balance these two choices to have a minimum risk from the seller's perspective?

To simplify the model, let us only consider the hedging strategies involving a series of stopping times. Ahn et al. (1998) discussed how options can be replicated with transactions costs in a general diffusion setting where a sequence of stopping times are specified to satisfy required convergence conditions. But, the convergence result assumes that transactions costs are equal to or greater than the square root of the size of rebalance intervals, which is unrealistic in practice. Two popular strategies are in this category. The first is the delta hedging strategy with deterministic time rebalancing, and the second is the delta hedging strategy with portfolio adjustments determined by the price move in the underlying security. Martellini and Priaulet (2002) compared the performance of these strategies. Grannan and Swindle (1996) and Toft (1996) studied the move based strategy (stopping time approach). Toft(1996) showed that strategies that are based on the moves in the underlying asset are generally superior to simple discretely rebalancing strategies.

Let $\tau_{1}, \ldots, \tau_{n}$ be all time epochs when hedging trades take place for a given horizon $T$. The number $n$ may be random depending on the outcomes of the underlying security prices. Denote $\tau_{n+1}=T$. For the Black-Scholes delta hedging strategy, the volatility of the underlying asset is required for the model input which is not observable. Leland (1985) used an augmented volatility in hopping to offset the hedging cost. This idea is useful in terms of measuring the influence of transaction costs.

Suppose the dynamics of the underlying security follows Equation (1). Let $\hat{\sigma}$ be the optimally adjusted volatility (to be decided later) for the delta hedging strategy, then the 
hedging portfolio dynamics $P_{\tau_{i}}$ follows the recurrence:

$$
\left\{\begin{array}{l}
P_{0}=(1-k) S_{0} N\left(\hat{d}_{0}\right)-K e^{-r T} N\left(\hat{d}_{0}-\hat{\sigma} \sqrt{T}\right) \\
P_{\tau_{i+1}}=S_{\tau_{i+1}} N\left(\hat{d}_{\tau_{i}}\right)+\left(P_{\tau_{i}}-S_{\tau_{i}} N\left(\hat{d}_{\tau_{i}}\right)\right) e^{r\left(\tau_{i+1}-\tau_{i}\right)}-k\left|N\left(\hat{d}_{\tau_{i+1}}\right)-N\left(\hat{d}_{\tau_{i}}\right)\right| S_{\tau_{i+1}}
\end{array}\right.
$$

where $P_{0}$ is equal to the adjusted option value minus the initial portfolio transaction costs, and

$$
\hat{d}_{\tau_{i}}=\frac{\ln \frac{S_{\tau_{i}}}{K}+\left(r+\frac{1}{2} \hat{\sigma}^{2}\right)\left(T-\tau_{i}\right)}{\hat{\sigma} \sqrt{T-\tau_{i}}} .
$$

The optimality of $\hat{\sigma}$ depends not only on the rate of the transaction cost $k$ but also on the way of triggering hedging trades. In the deterministic case, the optimal $\hat{\sigma}$ depends on the length of the rebalance interval. The finer the rebalance interval, the smaller the hedging error, but the larger the transactions costs. On the other hand, a move based oriented approach can avoid enormous trading. Investors can choose triggering limits in the change of the underlying stock returns for portfolio rebalancing. The following setting is used in our study. For $i=0,1, \ldots, n$.

$$
\tau_{i+1}=\min \left(T, \tau_{i}+\inf \left\{t>0 ; \ln \frac{S_{t+\tau_{i}}}{S_{\tau_{i}}} \geq u \text { or } \leq-l\right\}\right)
$$

where $\tau_{0}=0$ and $(l, u) \geq 0$ are the decrement and increment thresholds for rebalancing in terms of the rate of return in the underlying asset. The hedging trade is triggered when the growth of the underlying asset is found outside of the interval $[-l, u]$. The advantage of using a move-based hedging strategy is that the hedging strategy is "continuously" implemented. The agent can make hedging trades by observing the movements of the underlying security without missing any "jumps" that may result in any catastrophes as in the case of discretely rebalancing strategies. As in Leland (1985), we assume that the price of the option is given by the Black-Scholes formula with an adjusted volatility for the delta hedging throughout the horizon. That is, the price of the option is

$$
C_{0}=S_{0} N\left(\hat{d}_{0}\right)-K e^{-r T} N\left(\hat{d}_{0}-\hat{\sigma} \sqrt{T}\right) .
$$




\section{B. Hedging Error and the Optimization Model}

We have analyzed the hedging strategy and the hedging portfolio dynamics. Now we turn to the optimization model. Since exact hedging is not possible, we must find a suitable return/risk tradeoff. From the simulations conducted in Section I, delta hedging can exactly replicate the option's payoff if transaction costs are ignored. This indicates that mean absolute error of the replication approaches zero as the length of the rebalance interval tends to zero. This suggests that, given the available market trading environment and possible constraints, we may want to find out how close the hedging portfolio to the actual option's payoff. The investor has two components in the whole portfolio, a short position in the option contract and a long position in the hedging portfolio. For Black-Scholes Model, delta hedging achieves an exact replication of the option; both upside and downside errors are exactly equal to zero when trading takes place continuously. However, this is no more the case in the presence of transaction costs. Let $C_{T}=\left(S_{T}-K\right)^{+}$be the payoff of a call option with strike price $K$ and expiry date $T$ and $P_{T}$ the terminal portfolio value as defined in (7). The total replication error is

$$
\left|P_{T}-C_{T}\right|=\left(P_{T}-C_{T}\right)^{+}+\left(P_{T}-C_{T}\right)^{-}
$$

Since exact hedge with transaction costs is impossible, investors may view upside replication errors, $\left(P_{T}-C_{T}\right)^{+}$, differently from the downside replication errors, $\left(P_{T}-C_{T}\right)^{-}$. The call writer strives to minimize the downside replication losses while the upside replication gains must be limited. How does the investor rationally assign a "utility" function for the tradeoff between the two replication errors? Empirical evidence exhibits that the implied volatilities for equity options are heavily skewed, which suggests that risk is not symmetric and it dependents on the moneyness of the option. If the probability that the option expires in the money is large, investors then may be more concerned about the downside replication losses considering the issued option as a liability. Denote $\phi_{K}$ the probability that the option with strike price $K$ ends valueless at the expiry date, i.e.,

$$
\phi_{K}=\operatorname{Pr}\left[S_{T} \leq K\right]
$$


which implies that

$$
\phi_{K}=N\left(-\frac{\ln \left(S_{T} / K\right)+\left(\mu-\frac{1}{2} \sigma^{2}\right) T}{\sigma \sqrt{T}}\right) .
$$

The investor's objective is to minimize the weighted mean errors:

$$
\phi_{K} E\left[\left(P_{T}-C_{T}\right)^{+}\right]+\left(1-\phi_{K}\right) E\left[\left(P_{T}-C_{T}\right)^{-}\right] .
$$

If $E\left[\left(P_{T}-C_{T}\right)^{+}\right]$is considered as the overall performance for the favorable outcome and $E\left[\left(P_{T}-C_{T}\right)^{-}\right]$the overall performance for the unfavorable outcome, Equation (9) can be viewed as the expected performance using the probability measure induced by the moneyness of the option. Since the probability that the option is out of the money at expiration increases with the level of the strike prices, the interest in hedging the downside error decreases as the option strike price increases, which indicates that the investor prefers to charge a higher premium for deep in the money options and lower premium for deep out-of-the-money options than the Black Scholes options prices. For a given sequence of stopping times $\tau_{i}$ determined by the portfolio rebalancing policy, the writer of the call option must solve the following nonlinear optimization model

$$
\begin{array}{ll}
\min _{\hat{\sigma}>0} & \phi_{K} E\left[\left(P_{T}-C_{T}\right)^{+}\right]+\left(1-\phi_{K}\right) E\left[\left(P_{T}-C_{T}\right)^{-}\right] \\
\text {s.t. } & P_{0}=(1-k) S_{0} N\left(\hat{d}_{0}\right)-K e^{-r T} N\left(\hat{d}_{0}-\hat{\sigma} \sqrt{T}\right) \\
& P_{\tau_{i+1}}=S_{\tau_{i+1}} N\left(\hat{d}_{\tau_{i}}\right)+\left(P_{i}-S_{\tau_{i}} N\left(\hat{d}_{\tau_{i}}\right)\right) e^{r\left(\tau_{i+1}-\tau_{i}\right)}-k\left|N\left(\hat{d}_{\tau_{i+1}}\right)-N\left(\hat{d}_{\tau_{i}}\right)\right| S_{\tau_{i+1}} \\
& i=0,1, \ldots, n .
\end{array}
$$

The Black-Scholes option price is a special instance of the solution to the optimization model (9) if transaction costs are ignored. In fact, as the thresholds triggering hedging trades approach zero, which implies a continuous trading in the limit, both the upside replication gains and the downside replication losses must tend to zero, since exact hedge can be achieved in the framework of continuous trading without transaction costs. Thus, the optimization model (9) is consistent with Black-Scholes model. We will show a simulation result to illustrate this in the next section.

The optimization model (9) is difficult to solve. From the recurrence relation, the terminal 
portfolio value can be represented as

$$
P_{T}=P_{0} e^{r T}+\sum_{i=0}^{n}\left(S_{\tau_{i+1}} e^{r \tau_{i+1}}-S_{\tau_{i}} e^{r \tau_{i}}\right) N\left(\hat{d}_{\tau_{i}}\right)-k \sum_{i=0}^{n}\left|N\left(\hat{d}_{\tau_{i+1}}\right)-N\left(\hat{d}_{\tau_{i}}\right)\right| S_{\tau_{i+1}} e^{r \tau_{i+1}} .
$$

However, the closed form expression for the probability distribution function of the stopping time $\tau_{i}, i=1,2, \cdots, n$, is available only in a mathematical series. Nowadays, numerical solutions can be made as accurate as one dictates, exploiting a highly versatile software package. In this paper we use Matlab to complete such a task. All codes are available from the suthors upon request. First we simulate a large number of scenario paths of the underlying asset for given model parameters. Then, we apply a process control strategy to implementing portfolio hedging overtime, taking transaction costs into consideration. Several related questions may be asked. How should an investor set the two triggering thresholds? What is the expected number of portfolio adjustments? How big is the expected loss over the option's payoff? These quantities are reported in the next section when we apply the model to real data.

Instead of exogenously specifying an augmented volatility for the Black-Scholes formula, we endogenize the hedging volatility for the calculation of delta hedging. The simulation result excluding transaction costs is almost identical to the Black-Scholes approach which proves that this method is useful. The optimal value of the hedging volatility is determined by minimizing the "expected" hedging error ${ }^{2}$ at the expiry date of the call option. Our expectation is that the optimally obtained hedging volatility for the delta hedging strategy produces a close explanation for the phenomenon of volatility skew.

\footnotetext{
${ }^{2}$ This is different from the concept of mean absolute error. Actually, to reflect investors' preference, the mean absolute error is split into two quantities, the upside replication error and the downside replication error, which are weighted using the probability that the option is out of the money. This setting of the objective reflects that, the more favorable the option is, the less interested the investor would be in hedging the upside error.
} 


\section{Comparisons with Empirical Data}

Data on S\&P 500 cash options as presented in the beginning of Section II is used for the model input to make a close comparison between the simulation result and the actual outcome of the options' prices.

A puzzling phenomenon in the theory of equity option pricing is the skewness of implied volatilities. The Black-Scholes formula assumes constant volatility, hence, the prices of options with different strike prices should reflect that. But, as in Figure 3, the implied volatilities are not flat; the implied volatilities for deep in-the-money (out-of-the-money) options are much higher (lower) than the volatility of the underlying index. There might be different reasons; see e.g. Hodges, Tampkins and Ziemba (2002) for discussions and further results. We examine how transactions costs and investor's replication decision can explain this phenomenon.

Martellini and Priaulet (2002) found that, for all strategies, the higher the volatility, the higher the transaction costs. The intuition is that, when the hedging volatility increases, dynamic replication of the option's payoff involves more transactions. How do we avoid this disadvantage? A move-based strategy can balance the hedging error and the transaction cost. If the hedging strategy based on an optimally chosen "volatility" is jointly used with preset triggering rules for portfolio adjustments, the option can be efficiently replicated and the option price based on the hedging portfolio value can be more practical.

As before, we set the transaction cost $k=0.1 \%$. Observations of the index levels are on a daily basis. With the thresholds ${ }^{3}, l=u=1 \%$, the expected number of portfolio adjustments is approximately 54, meaning the number of days between two consecutive adjustments is about 2 to 3 . The simulation and the optimization models are for all strike prices from 1025

\footnotetext{
${ }^{3}$ We can also endogenize these parameters in the optimization model. The optimal thresholds may differ with the size of the transaction costs. It is conceivable that, the greater the transaction costs, the larger the optimal thresholds might be to have a good balance between the total transaction cost and the hedging error. Adding these variables brings about much complexity to the model. Without losing the points, we set these parameters exogenously.
} 
to 1350 . Table III presents the results.

$$
<<\text { Table III about here }>>
$$

For this period, the Black-Scholes formula tends to underprice the in-the-money options and overprice the out-of-the-money options. However, the prices (third column) by hedging with transaction costs appear to be higher for in-the-money options and lower for out-ofthe-money option than the Black-Scholes prices (sixth column). Furthermore, the hedging volatilities (fifth column) are very consistent with the empirical foundings. Figure 4 depicts how close the optimal hedging volatilities are to the actual implied volatilities. The optimal hedging volatilities are slightly lower (higher) than the implied volatilities for deep in-themoney (out-of-the-money) call options, while they are close to each other for call options that are around at-the-money.

While options' prices obtained using the suggested model are quite close to the actual prices, it is interesting to examine the replication differences, which is defined as the difference between the portfolio terminal value and the option's payoff, $P_{T}-C_{T}$. Figures 547 show the hedging errors for deep in-the-money options, at the money options, and deep out-of-themoney options, respectively.

$$
<<\text { Figures } 5.7 \text { about here }>>
$$

There is still quite a substantiate amount of short hedging for deep in-the-money options, which reflects that the option premiums are too low. For the at-the-money options, the hedging difference has a mean zero. The hedging difference is most likely positive, indicating that out-of-the money options' premiums are too high. These findings support a strong empirical skewness of option implied volatilities.

Why does the magnitude of the hedging difference range so much? This is due to the setting of time frame of the hedging. The calculation of the delta at the time close to the expiration date is very sensitive to the ratio of the underlying asset and the option strike price, though the delta at expiration time is theoretically either 0 or 1 depending on the 
outcome. However, if the underlying asset ends around the strike price, the delta for the final portfolio adjustment can be any number within $[0,1]$, depending on the time partition. That is why the hedging difference is so volatile.

The investor may have a different view about the hedging difference. We now examine the upside and downside hedging errors for options with a range of strike prices. We expect to explain that the difference of options' premiums from the actual option prices are due to the upside and downside hedging errors. Figures 8 and 9 depict the upside and downside hedging errors for a deep in-the-money option (with strike $K=1025$ ).

$$
<<\text { Figures } 8 \text { and } 9 \text { about here }>>
$$

For deep in-the-money options, the upside hedging errors are relatively small, while the downside hedging errors are large, indicating a higher option premium should be required. Figures 10 and 11 depict the upside and downside hedging errors for the at-the-money option (with strike $K=1150$ ).

$$
<<\text { Figures } 10 \text { and } 11 \text { about here }>>
$$

For the at-the-money options, both hedging errors and downside errors are not obviously different one from the other. There is not much difference between the model implied value and the actual option price. Since for at-the-money option, the probability of losing money is about 0.5 , which indicates that the investor is indifferent between the upside replication errors and the downside ones. Figures 12 and 13 depict the upside and downside hedging errors for a deep out-of-the-money option (with strike $K=1350$ ).

$$
<<\text { Figures } 12 \text { and } 13 \text { about here }>>
$$

Contrary to the case of deep in-the-money options, for deep out-of-the-money options, the downside hedging errors appear relatively small, while the upside hedging errors are large, indicating a lower option premium should be required. 
The Black-Scholes option price formula is a special instance when transaction costs are ignored. The mathematical proof is obvious as we argued briefly. It is interesting to demonstrate how close such a replication procedure can be made by choosing suitable length between security observation and by specifying arbitrarily small triggering thresholds for rebalancing. Table IV lists the simulation results with $l=u=0.1 \%$ and the time between two consecutive observations equal to $\frac{1}{1024}$. Other parameters are the same as before.

$$
<<\text { Table IV about here }>>
$$

The expected number of portfolio adjustments is 825 (approximately 6 times a day). For the given rebalance strategy, the difference between the hedging portfolio and the option's payoff are kept within the range from -0.09 to -1.19 . The optimal hedging volatilities range from 0.1777 to 0.1886 . As the rebalance interval and the thresholds approach zero, the optimal solution to model (9) converges to the Black-Scholes option prices, which implies that the optimal $\hat{\sigma}$ is equal to the volatility of the underlying security for all levels of strike prices.

\section{Conclusion}

In the last thirty years, financial derivatives have grown from a marginal activity to occupy center-stage position in financial economic theory and financial practice. At the same time, mathematical finance has grown to be one of the main branches of applied mathematics. The single largest credit for these remarkable developments are due to Fisher Black, Myron Scholes, and Robert Merton, who's classic 1973 papers gave a theory of how to price options. Without this prescription, option pricing would have remained more of an art than a science, and trading in options would have been less liquid and less important, as traders would have had a less firm idea on how to fairly value and hedge the options. However, this great achievement rests on the assumptions of no arbitrage, lognormality for spot price dynamics, and frictionless trading. In reality, even though the condition of arbitrage free and the assumption of lognormality are arguably to be satisfactory most of the time, transaction costs always exist. 
Option sellers have to take transaction costs as an influential factor on the option's pricing and hedging. There have been several empirical and theoretical modifications to the Black-Scholes theory. Boyle and Vorst (1992) and Leland (1985) are two seminal papers on option hedging with transaction costs. The first extends Cox, Ross and Rubinstein (1979) model and the latter extends the Black-Scholes model with transaction costs. Although the theorem in Leland (1985) is flawed, the idea is still useful.

Based on the Black-Scholes setting, we have presented a simple model based on a risk management objective. With empirical data, option price modelling approach suggested in this paper provides an explanation of volatility skewness for equity options. 


\section{References}

Ahn, Hyungsok, Mohit Dayal, Eric Grannan, and Glen Swindle, 1998, Option replication with transaction costs: General diffusion limits, Annals of Applied Probability 8, 676-707.

Bensaid, B., J.P. Lesne, H. Pages, and J. Scheinkman, 1992, Derivative asset pricing with transaction costs, Mathematical Finance 2, 63-86.

Black, F., and M Scholes, 1973, The pricing of options and corporate liabilities, Journal of Political Economy 81, 637-654.

Boyle, P.P., and T. Vorst, 1992, Option replication in discrete time with transactions costs, Journal of Finance 47, 271-293.

Constantinides, Geroge, and Thaleia Zariphopoulou, 1999, Bounds on prices of contingent claims in an intertemporal economy with proportional transaction costs and general preference, Finance and Stochastics 3, 345-369.

— , 2001, Bounds on prices of contingent claims in an intertemporal economy with multiple securities, Mathematical Finance 11, 331-346.

Cox, J., S. Ross, and M. Rubinstein, 1979, Option pricing: A simplified approach, Journal of Financial Economics 7, 229-263.

Davis, M.H.A., and J.M.C. Clark, 1994, A note on super replicating strategies, Phil. Trans. Roy. Soc. London Ser. A347, 485-494.

Davis, M.H.A., V.G. Panas, and T. Zariphopoulou, 1993, European option pricing with transaction costs, SIAM Journal on Control and Optimization 31, 470-493.

Edirisinghe, C., V. Naik, and R. Uppal, 1993, Optimal replications of options with transactions costs, Journal of Financial and Quantitative Analysis 28, 117-138.

Grannan, E.R., and G.H. Swindle, 1996, Minimizing transaction costs of option hedging strategies, Mathematical Finance 6, 341-364. 
Hodges, Stewart D., Robert G. Tompkins, and William T. Ziemba, 2002, The favorite longshot bias in S\&P 500 futures options: The return to bets and the cost of insurance, working paper.

Hoggard, T., A.E. Whalley, and P. Wilmott, 1994, Hedging option portfolios in the presence of transaction costs, Advances in Futures and Options Research 7, 21-35.

Kabanov, Yuri M., and Mher M. Safarian, 1997, On Leland's strategy of option pricing with transaction costs, Finance and Stochastics 1, 239-250.

Leland, H.E., 1985, Option pricing and replication with transaction costs, Journal of Finance 40, 1283-1301.

Martellini, Lionel, and Philippe Priaulet, 2002, Competing methods for option hedging in the presence of transaction costs, Journal of Derivatives 2, 26-38.

Merton, Robert C., 1973, Theory of rational option pricing, The Bell Journal of Economics and Management Science 4, 141-183.

Rubinstein, Mark, 1994, Implied binomial trees, Journal of Finance 49, 771-818.

Soner, H.M., S. Shreve, and J. Cvitanic̀, 1995, There is no nontrivial hedging portfolio for option pricing with transaction costs, Annals of Applied Probability 5, 327-355.

Toft, K.B., 1996, on the mean-variance tradeoff in option replication with transaction costs, Journal of Financial and Quantitative Analysis 31, 233-263. 


\section{Table I: Differences between Hedging Portfolios and Option Payoffs at the Expiration (1 Year) for Different Rebalance Intervals}

$(1 / 260,1 / 520,1 / 1040,1 / 2080,1 / 4160,1 / 8320)$

Strike Price

Option Value

Mean

Standard Deviation

Mean

Standard Deviation

Mean

Standard Deviation

Mean

Standard Deviation

Mean

Standard Deviation

Mean

Standard Deviation

$\begin{array}{rrrrr}80 & 90 & 100 & 110 & 120 \\ 25.4125 & 18.1408 & 12.3360 & 8.0264 & 5.0254\end{array}$

Rebalance Interval $=1 / 260$

$\begin{array}{lllll}0.0247 & 0.0335 & 0.0251 & 0.0111 & 0.0085 \\ 0.2939 & 0.4406 & 0.5233 & 0.5905 & 0.6320\end{array}$

Rebalance Interval $=1 / 520$

$\begin{array}{lllll}0.0168 & 0.0219 & 0.0083 & 0.0156 & 0.0095\end{array}$

$\begin{array}{lllll}0.2007 & 0.3042 & 0.3660 & 0.4110 & 0.4129\end{array}$

Rebalance Interval $=1 / 1040$

$\begin{array}{lllll}0.0074 & 0.0074 & 0.0083 & 0.0156 & 0.0095\end{array}$

$\begin{array}{lllll}0.1419 & 0.2056 & 0.2684 & 0.3025 & 0.2911\end{array}$

Rebalance Interval $=1 / 2080$

$\begin{array}{rrrrr}-0.0031 & -0.0069 & -0.0036 & -0.0003 & -0.0008 \\ 0.0957 & 0.1443 & 0.1887 & 0.2066 & 0.2137\end{array}$

Rebalance Interval $=1 / 4160$

$\begin{array}{lllll}0.0027 & 0.0017 & 0.0013 & 0.0070 & 0.0036 \\ 0.0696 & 0.1057 & 0.1338 & 0.1498 & 0.1552\end{array}$

Rebalance Interval $=1 / 8320$

$\begin{array}{rrrrr}0.0014 & 0.0017 & 0.0036 & 0.0087 & -0.0053 \\ 0.0545 & 0.0780 & 0.0946 & 0.1049 & 0.1073\end{array}$




\section{Table II: Differences between Hedging Portfolios and Option Payoffs at the Expiration (1 Year) for Different Rebalance Intervals $(1 / 260,1 / 520,1 / 1040,1 / 2080,1 / 4160,1 / 8320)$-- Leland's Strategy}

Transaction Cost

Strike Price

Option Value
Mean
Standard Deviation
Option Value
Mean
Standard Deviation
Option Value
Mean
Standard Deviation
Option Value
Mean
Standard Deviation

Option Value

Mean

Standard Deviation

Option Value
Mean
Standard Deviation

$\begin{array}{rrrrr}0.001 & 0.001 & 0.001 & 0.001 & 0.001 \\ 80 & 90 & 100 & 110 & 120\end{array}$

Rebalance Interval $=1 / 260$, Adjusted Volatility $=0.2564$

$\begin{array}{rrrrr}25.5350 & 18.3334 & 12.5764 & 8.2794 & 5.2597 \\ -0.1819 & -0.2336 & -0.2845 & -0.3124 & -0.2982 \\ 0.3077 & 0.4533 & 0.5380 & 0.6092 & 0.6551\end{array}$

Rebalance Interval $=1 / 520$, Adjusted Volatility $=0.2589$

$\begin{array}{rrrrr}25.5859 & 18.4124 & 12.6744 & 8.3825 & 5.3555 \\ -0.2328 & -0.3217 & -0.4029 & -0.4189 & -0.4038 \\ 0.2292 & 0.3345 & 0.4039 & 0.4425 & 0.4493\end{array}$

Rebalance Interval $=1 / 1040$, Adjusted Volatility $=0.2626$

$\begin{array}{rrrrr}25.6579 & 18.5231 & 12.8112 & 8.5265 & 5.4897 \\ -0.3513 & -0.4499 & -0.5466 & -0.5767 & -0.5522 \\ 0.2094 & 0.2722 & 0.3291 & 0.3678 & 0.3840\end{array}$

Rebalance Interval $=1 / 2080$, Adjusted Volatility $=0.2676$

$\begin{array}{rrrrr}25.7599 & 18.6780 & 13.0016 & 8.7268 & 5.6772 \\ -0.4064 & -0.5945 & -0.7388 & -0.8049 & -0.7856 \\ 0.2499 & 0.2982 & 0.3254 & 0.3533 & 0.3948\end{array}$

Rebalance Interval $=1 / 4160$, Adjusted Volatility $=0.2745$

$\begin{array}{rrrrr}25.9042 & 18.8937 & 13.2651 & 9.0040 & 5.9378 \\ -0.5441 & -0.8166 & -1.0315 & -1.1114 & -1.0695 \\ 0.3108 & 0.3594 & 0.3790 & 0.4140 & 0.4821\end{array}$

Rebalance Interval $=1 / 8320$, Adjusted Volatility $=0.2841$

$\begin{array}{rrrrr}26.1079 & 19.1924 & 13.6269 & 9.3845 & 6.2977 \\ -0.7486 & -1.1160 & -1.3809 & -1.4951 & -1.4584 \\ 0.4285 & 0.4923 & 0.4862 & 0.5362 & 0.6531\end{array}$


Table III: Simulation Results $(l=u=1 \%, k=0.1 \%$ )

\begin{tabular}{cccccc} 
Strike $K$ & Actual Price & Implied Vol. & Hedging Price $\left(C_{0}\right)$ & Hedging Vol. $(\hat{\sigma})$ & B-S Price \\
\hline 1025 & 152.3 & 0.21906 & 151.1 & 0.21362 & 144.96 \\
1050 & 133 & 0.21485 & 131.56 & 0.20907 & 125.59 \\
1075 & 114.5 & 0.20958 & 113.15 & 0.20464 & 107.65 \\
1100 & 97.1 & 0.20409 & 96.593 & 0.20237 & 91.26 \\
1125 & 81.4 & 0.19994 & 81.098 & 0.19897 & 76.498 \\
1150 & 66.6 & 0.19415 & 67.306 & 0.19634 & 63.4 \\
1175 & 53.6 & 0.18931 & 54.761 & 0.19289 & 51.948 \\
1200 & 42.5 & 0.18552 & 44.249 & 0.19103 & 42.083 \\
1225 & 32.2 & 0.1792 & 34.991 & 0.18842 & 33.709 \\
1250 & 23.9 & 0.17418 & 27.273 & 0.18621 & 26.703 \\
1275 & 18 & 0.17266 & 20.823 & 0.18381 & 20.923 \\
1300 & 13.3 & 0.17116 & 15.064 & 0.17914 & 16.22 \\
1325 & 9.3 & 0.16784 & 10.821 & 0.17598 & 12.443 \\
1350 & 6.3 & 0.16459 & 7.5402 & 0.17273 & 9.4498
\end{tabular}


Table IV: Black Scholes Formula as a Special Instance

\begin{tabular}{ccccc} 
Strikes $K$ & B-S Prices & Hedging Prices $\left(C_{0}\right)$ & Hedging Volatilities $(\hat{\sigma})$ & Hedging Difference \\
\hline 1025 & 144.9628 & 145.8303 & 0.1886 & -0.0950 \\
1050 & 125.5943 & 126.3028 & 0.1872 & -0.1678 \\
1075 & 107.6516 & 108.1167 & 0.1860 & -0.2725 \\
1100 & 91.2595 & 91.7322 & 0.1858 & -0.3214 \\
1125 & 76.4982 & 76.6549 & 0.1847 & -0.4965 \\
1150 & 63.3997 & 63.3721 & 0.1841 & -0.6593 \\
1175 & 51.9479 & 51.6578 & 0.1833 & -0.8285 \\
1200 & 42.0831 & 41.4769 & 0.1823 & -0.9568 \\
1225 & 33.7092 & 32.8479 & 0.1814 & -1.0743 \\
1250 & 26.7029 & 25.6813 & 0.1806 & -1.1987 \\
1275 & 20.9228 & 19.8254 & 0.1799 & -1.1426 \\
1300 & 16.2196 & 14.9351 & 0.1786 & -1.1953 \\
1325 & 12.4432 & 11.3538 & 0.1787 & -0.9795 \\
1350 & 9.4498 & 8.3489 & 0.1777 & -0.9810
\end{tabular}




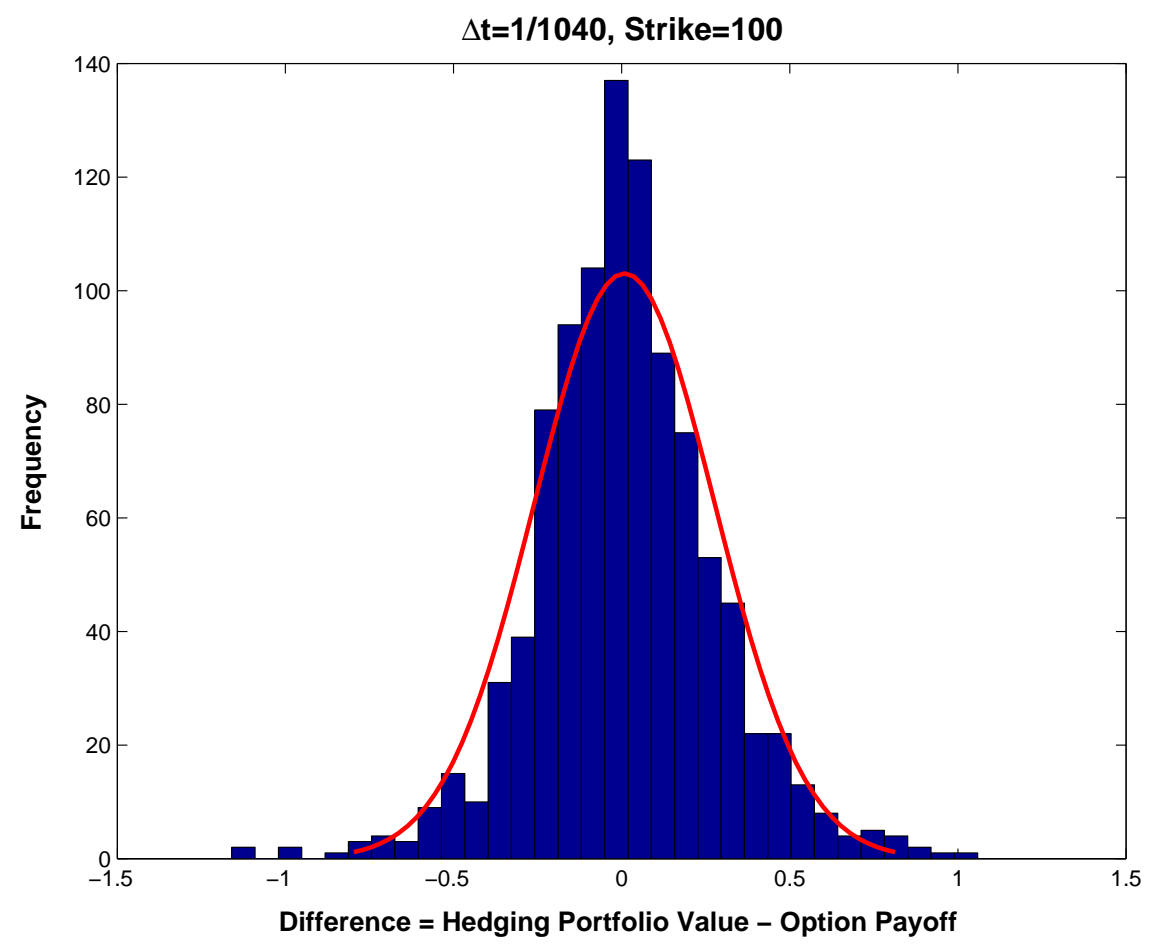

Figure 1. Distribution of the Total Portfolio Value without Transaction Costs

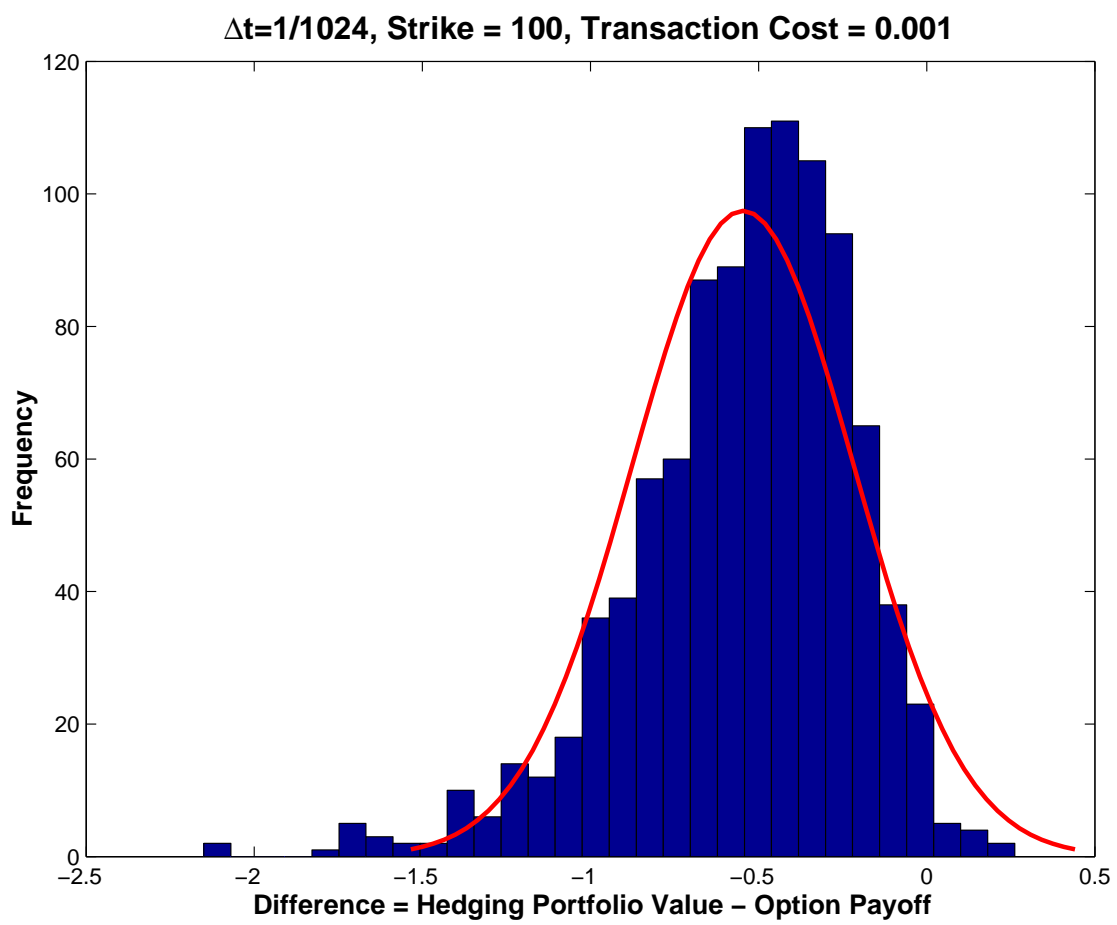

Figure 2. Distribution of the Total Portfolio Total Value with Transaction Costs 


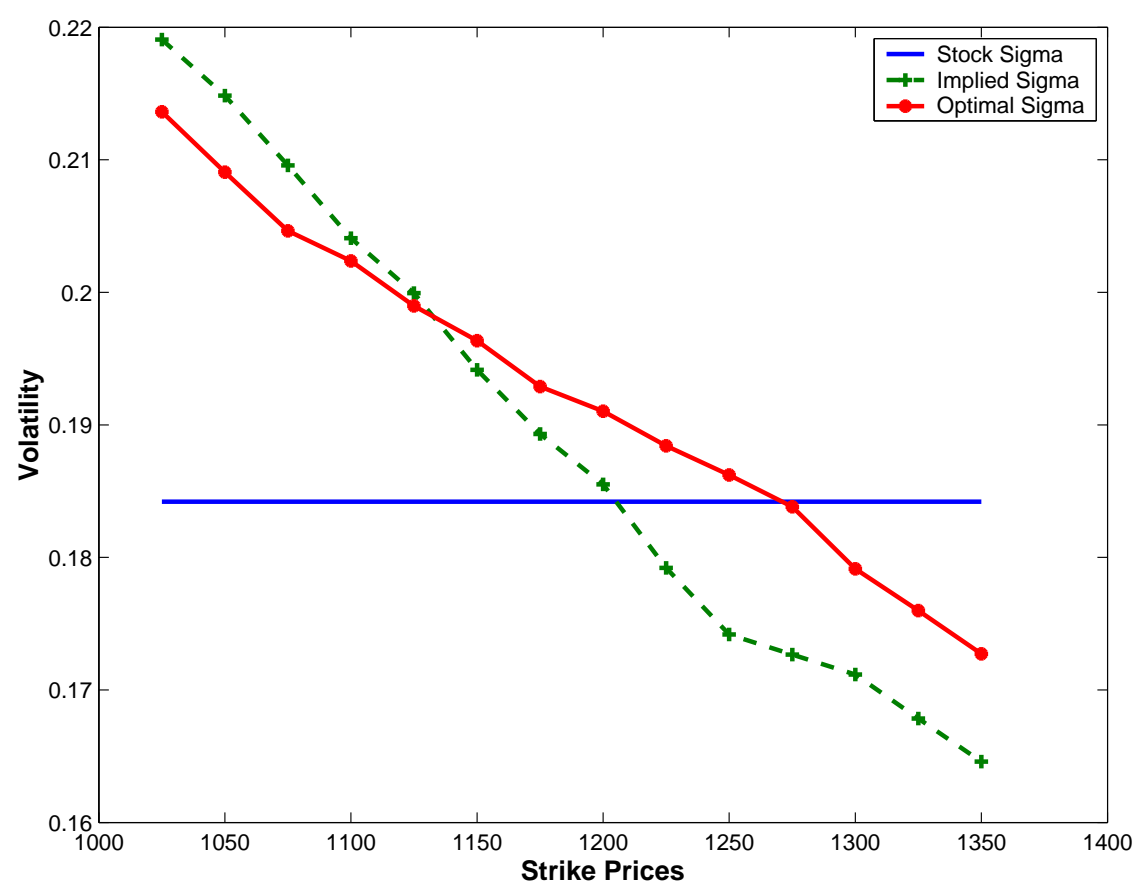

Figure 3. Implied and Hedging Volatilities across Strike Prices

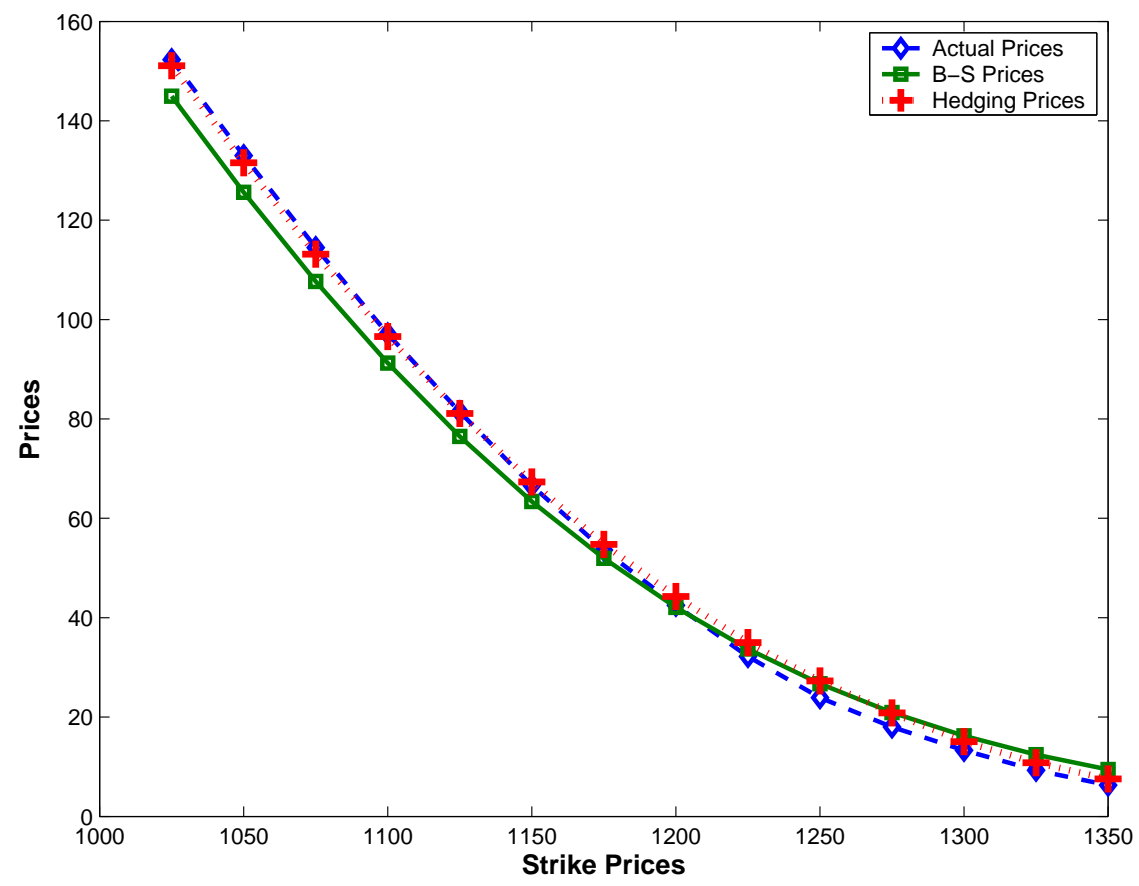

Figure 4. Actual, Black-Scholes, and Hedging Prices across Strike Prices 


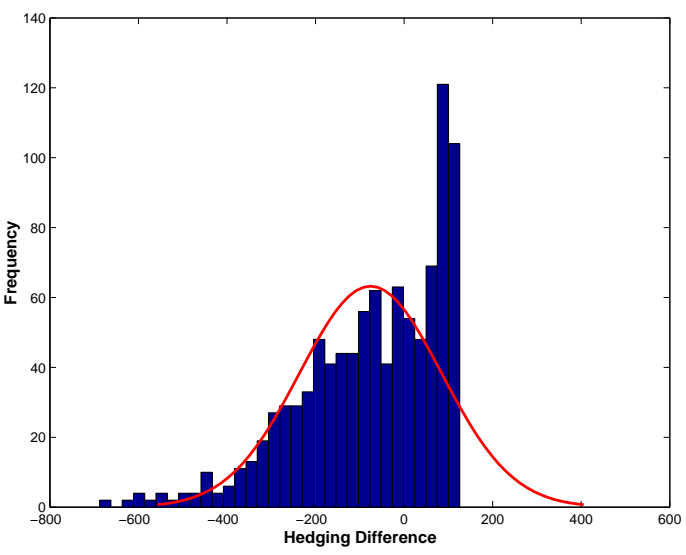

Figure 5. Hedging Differences for Deep In-The-Money Options

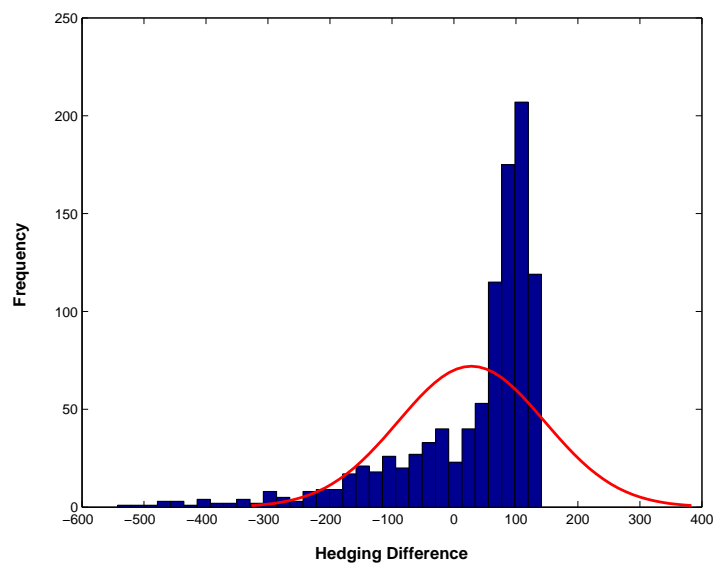

Figure 6. Hedging Differences for At-The-Money Options

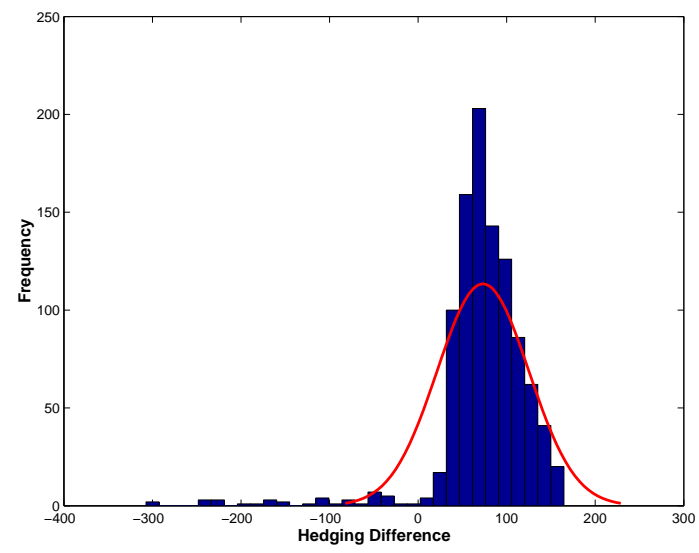

Figure 7. Hedging Differences for Deep Out-Of-The-Money Options 


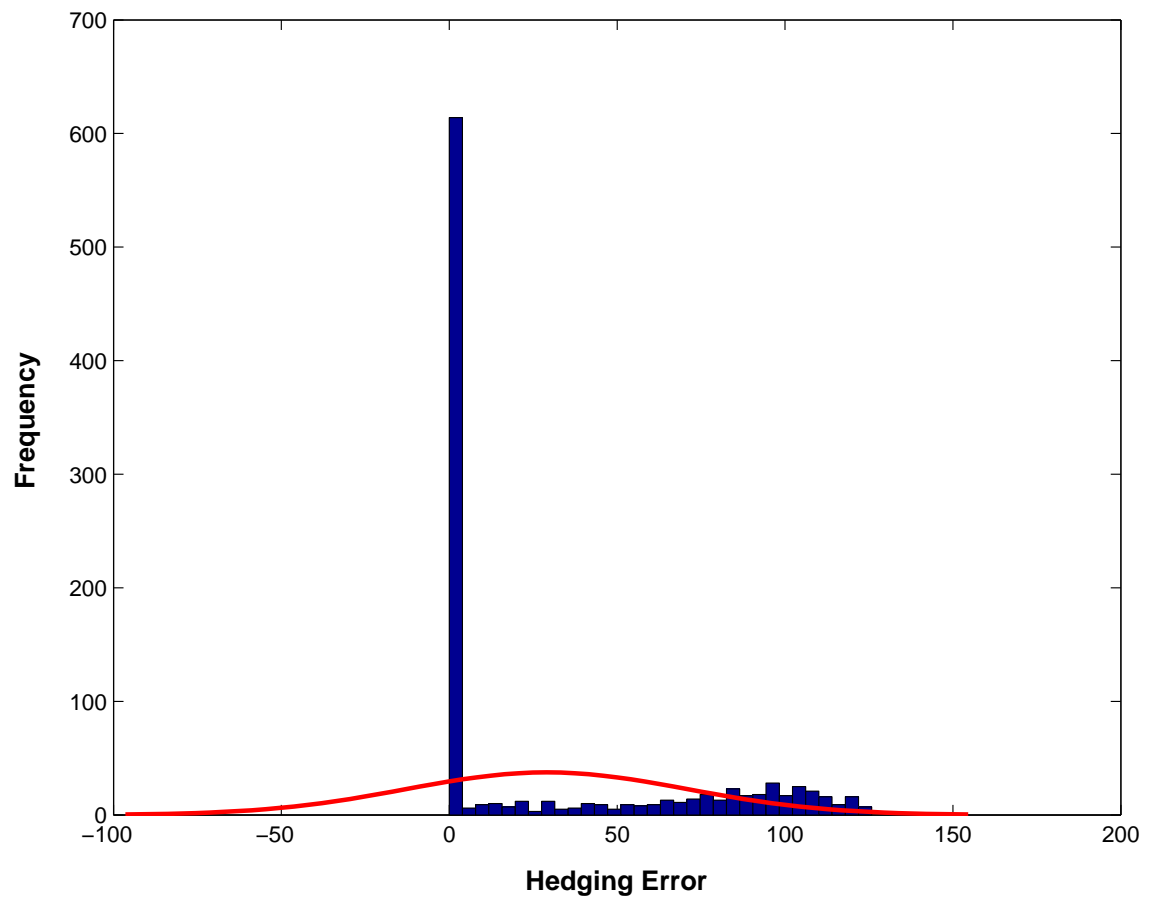

Figure 8. Upside Hedging Errors for Deep In-The-Money Options

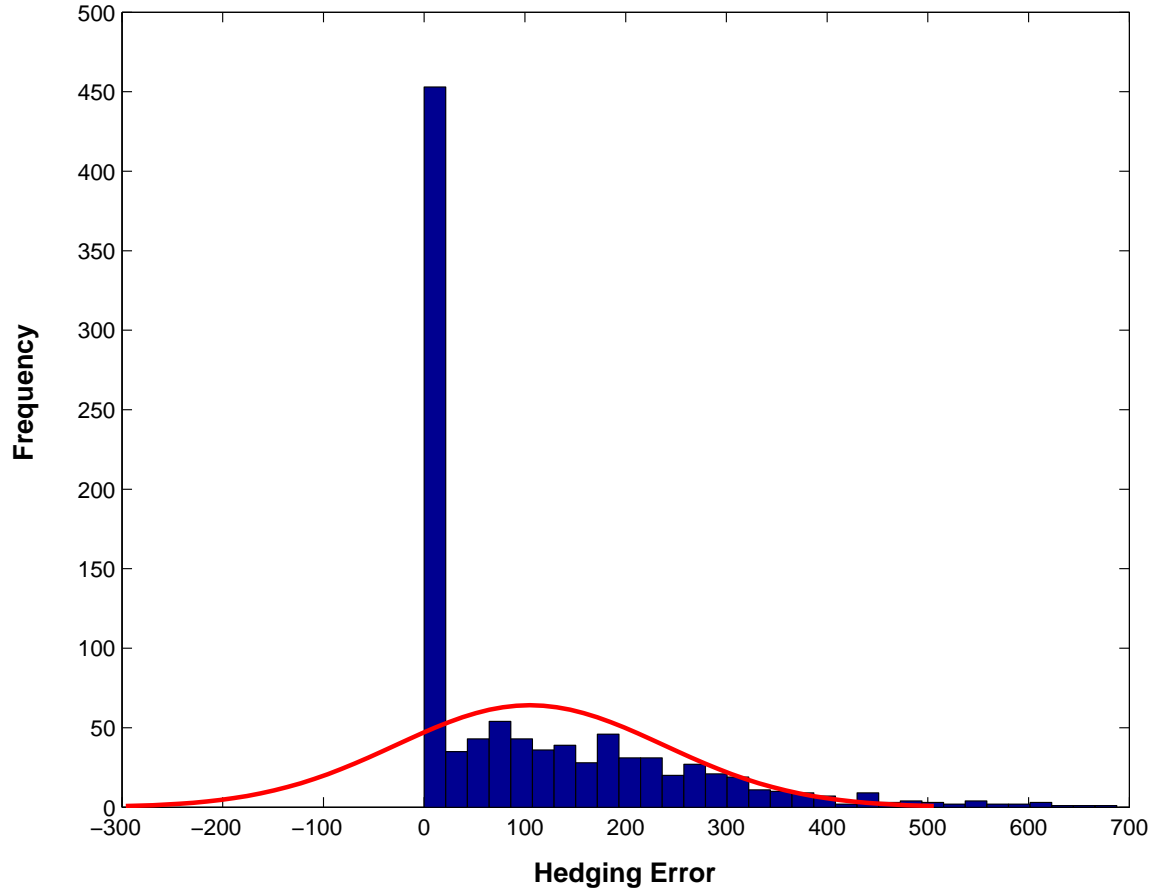

Figure 9. Downside Hedging Errors for Deep Out-Of-The-Money Options 


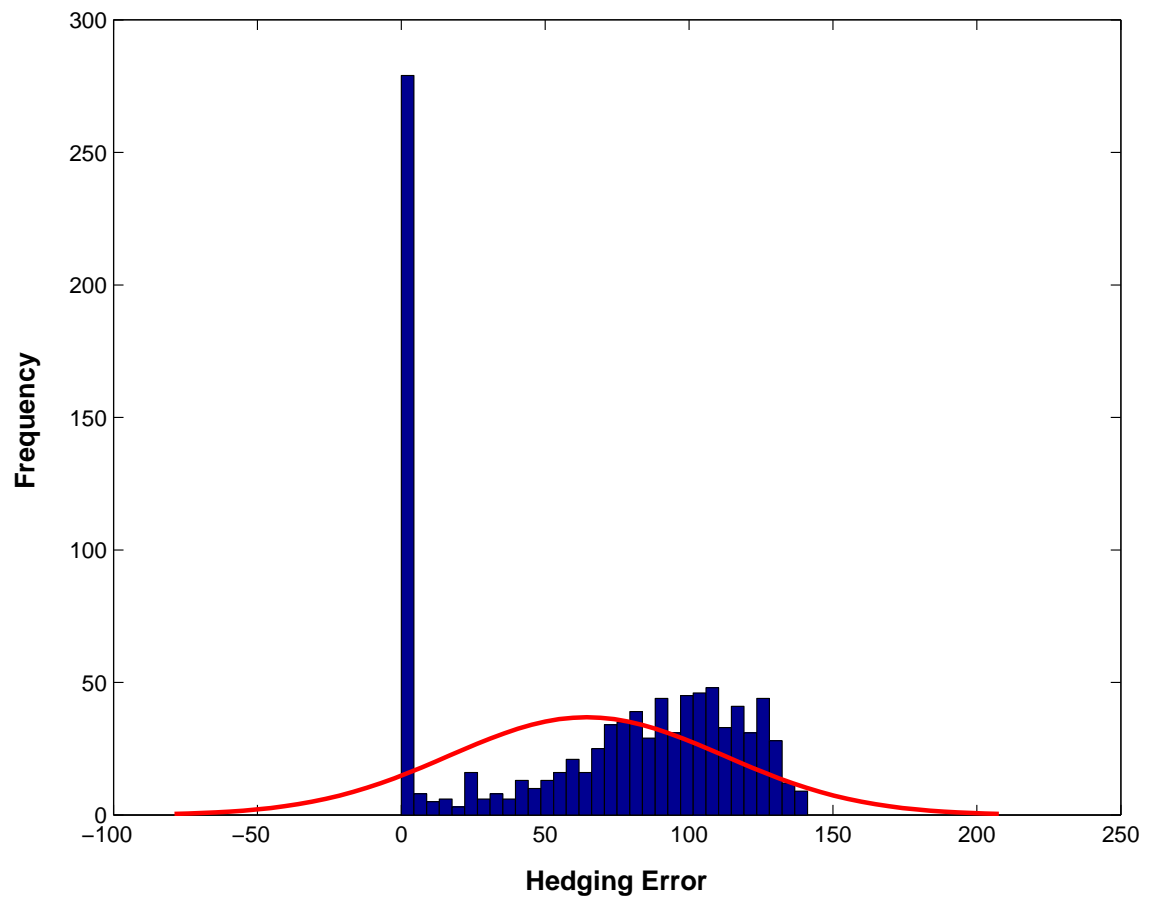

Figure 10. Upside Hedging Errors for the At-The-Money Option

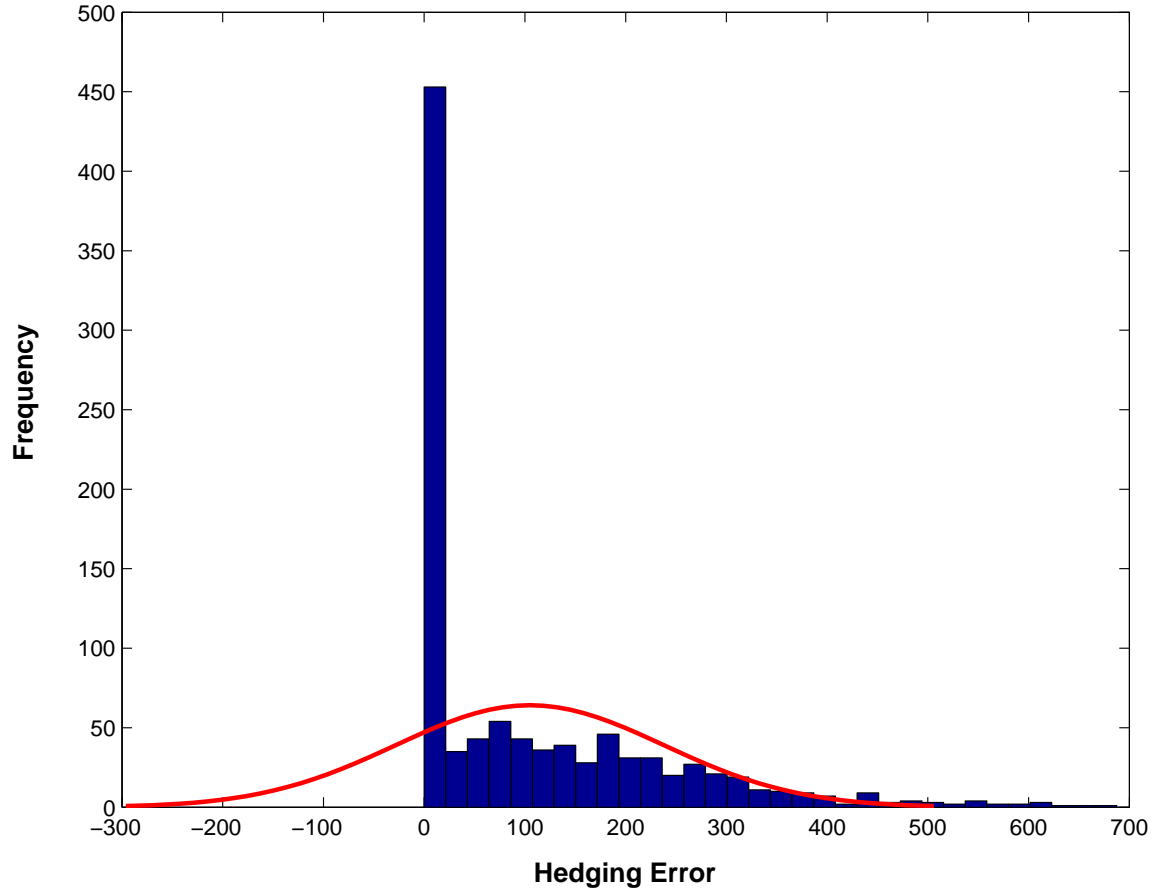

Figure 11. Downside Hedging Errors for the At-The-Money Option 


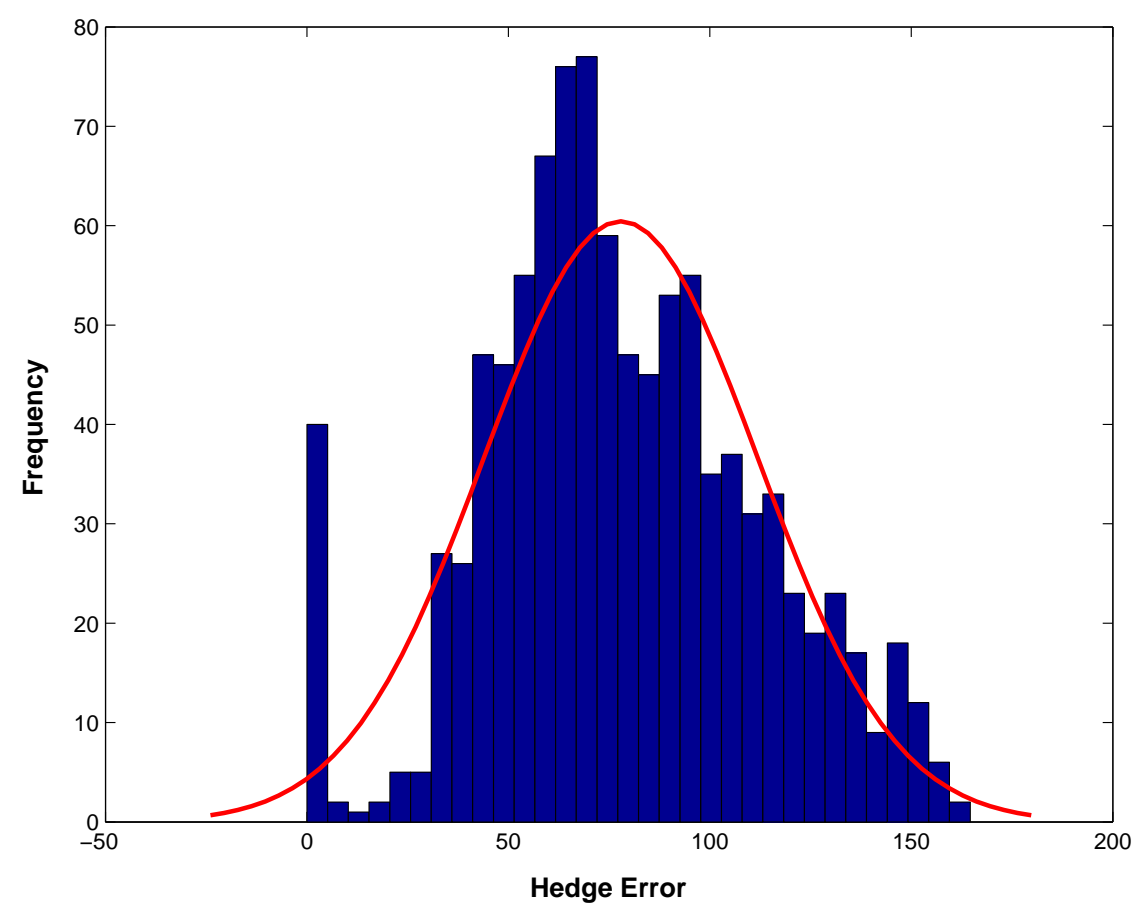

Figure 12. Upside Hedging Errors for a Deep Out-Of-The-Money Option

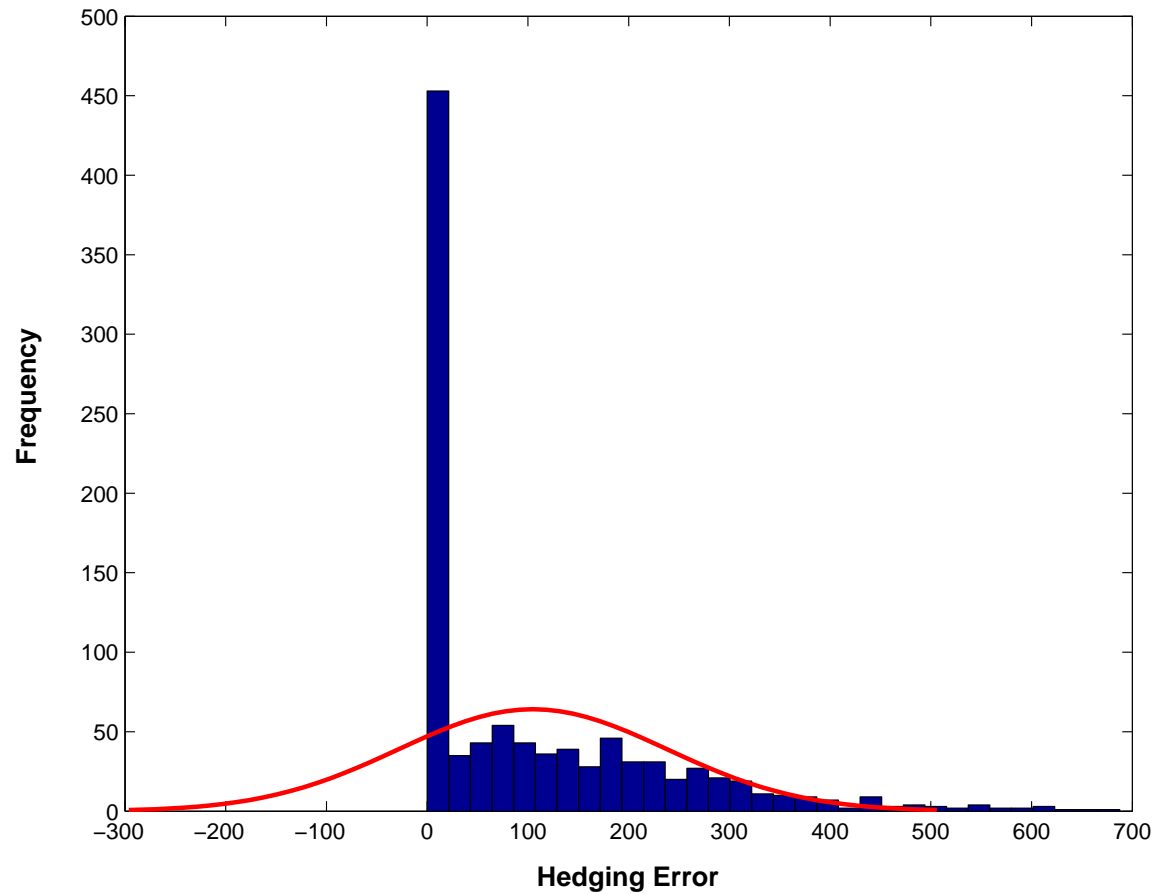

Figure 13. Downside Hedging Errors for a Deep Out-Of-The-Money Option 